\title{
Mathematical modeling of the magnetoelectric effect of the nano bi- layer L-T mode bar structure in high frequency regime
}

\author{
Treetep Saengow $^{1} \cdot$ Salinee Choowitsakunlert ${ }^{1} \cdot$ Rardchawadee Silapunt $^{1}$ (])
}

Received: 20 November 2020 / Accepted: 25 November 2020/ Published online: 27 May 2021

(C) The Author(s) 2021

\begin{abstract}
This paper describes the magnetoelectric (ME) coupling behavior of the nano bi-layer L-T (longitudinal-transverse) mode bar structure through the ME coefficient mathematical model that is developed in high frequency regime. Terfenol-D and Lead Zirconate Titanate (PZT) are used as ferromagnetic (FM) and ferroelectric (FE) layers, respectively. The ME coefficients are determined at different layer thickness ratios and products of the operating frequency $(f)$ and structure length $(l)$. It is found that the ME coefficient and optimal thickness ratio increases and decreases exponentially respectively, with $f$. The minimum and maximum peak ME coefficients at $f$ values of 0.1 and 1,200 respectively, are around 1,756 and $5,617 \mathrm{mV} / \mathrm{Oe} \cdot \mathrm{cm}$ with the optimal thickness ratio of 0.43 and 0.19 , respectively. The ME coupling behavior depends largely on the magnetostrictive effect in the FM layer that is altered by the applied magnetic field and $f$. The demonstration as the read sensor for the hard disk drive (HDD) with $2 \mathrm{Tbit} / \mathrm{in}^{2}$ areal density and 190 Oe/bit applied magnetic field shows the output voltage across the FE layer of around $0.43 \mathrm{mV}$, which is more than sufficient for the raw signal readback.
\end{abstract}

\section{Introduction}

Research on magnetoelectric (ME) materials has been growing dramatically owing to the material's potential for high frequency applications such as magnetic sensing in memory or storage devices and current-to-voltage conversion Vopsaroiu et al. (2007); Palneedi et al. (2006); Choowitsakunlert et al. (2014); Nan et al. (2008). The ME material is made of multiferroic composites that possess direct and converse ME coupling effects, which are the induction of electric polarization by the magnetic field and the induction of magnetization by the electric field, respectively Palneedi et al. (2006); Saengow et al. (2018); Wang et al. (2010). The ME material can be formed as a single-phase structure or a heterostructure. The heterostructure consists of alternate layers of ferromagnetic

Rardchawadee Silapunt

rardchawadee.sil@kmutt.ac.th

Treetep Saengow

treetep.marksaengow@mail.kmutt.ac.th

Salinee Choowitsakunlert

salinee.cho@mail.kmutt.ac.th

$1 \quad$ Electronic and Telecommunication Engineering, King Mongkut's University of Technology Thonburi, 126 PrachaUthit Rd., Thung Kru, Bangkok, Thailand
(FM) and ferroelectric (FE) materials, which exhibit magnetostrictive and piezoelectric properties, respectively. The ME coupling behaviour of an ME structure is commonly analyzed by a parameter known as ME coefficient. The ME coefficient is a measure of the ability of an ME material to generate the electric field from the input magnetic field and vice versa.

There were several studies on developing mathematical models of the ME coefficient of the nano heterostructures with different input-output field orientations and operating modes such as L-T (longitudinal-transverse) and T-T (transverse-transverse) modes with bar and plate structures, respectively Vopsaroiu et al. (2007); Saengow et al. (2018); Wang et al. (2010); Saengow and Silapunt (2020). These models were developed under the low frequency $(\mathrm{f} \rightarrow 0)$ approach, to reduce complexity of the modeling Vopsaroiu et al. (2007); Saengow et al. (2018). It was shown that that the L-T mode bar structure exhibits higher ME coefficient and offers more flexibility in the structure design, compared to the plate structure Saengow and Silapunt (2020). The low frequency approach, however, shadows the actual ME coupling behaviour of the defined structure.

This paper is aimed on understanding the actual ME coupling behaviour of the nano bi-layer L-T mode bar structure through the ME coefficient mathematical model 
that is developed in high frequency regime. The ME coefficient model will then be tested on the bi-layer of Terfenol-D and lead zirconate titanate (PZT). The magnetostrictive effect that defines the ME mechanism in the proposed structure will be discussed. Finally, the demonstration of the proposed structure as the nano read sensor for a hard disk drive is performed. The readout voltage is determined and the feasibility of the structure for the magnetic sensing application is assessed.

\section{Mathematical modeling of the magneto electric coefficient of the nano bi-layer L-T mode bar structure}

The MF composites of the nano-bilayer in a shape of bar structure is considered with length $(l)$, width $(w)$, and total thickness $(t)$ as shown in Fig. 1. Note that, the design condition is $l \gg w, t$ Vopsaroiu et al. (2007). The magnetostrictive constitutive equation, piezoelectric constitutive equation, and Newton's second law are employed altogether for the modeling of the ME coefficient of the proposed structure.

Magnetostrictive constitutive equation is a relationship between magnetic field, stress, and strain as shown in Eq. (1). For the L-T mode, the external magnetic field is applied to the FM layer in a longitudinal direction in order to induce the mechanical strain in the same direction. This strain is then coupled to the FE layer.

$\chi_{1}^{m}=s_{11}^{m} \sigma_{1}^{m}+d_{11}^{m} H_{1}$,

where $\chi$ is strain, $s$ is elastic compliance, $\sigma$ is stress, $d^{\mathrm{m}}$ is the ferromagnetic constant, and $H$ is magnetic field. Superscript ' $m$ ' refers to a ferromagnetic material and subscript ' 1 ' refers to the longitudinal direction.

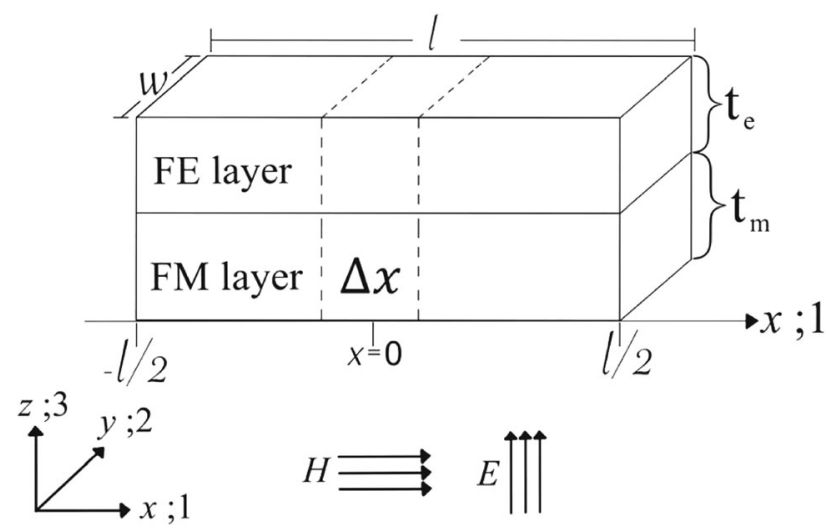

Fig. 1 Multiferroic composites of the nano bi-layer L-T mode bar structure
The piezoelectric constitutive equation is used to explain the relationship between stress, strain and electric field as shown in Eq. (2) and between stress, electric field, and electric displacement as shown in Eq. (3).

$\chi_{1}^{e}=s_{11}^{e} \sigma_{1}^{e}+d_{31}^{e} E_{3}$

$D_{3}=d_{31}^{e} \sigma_{1}^{e}+\epsilon_{33} E_{3}$,

where $d^{\mathfrak{e}}$ is piezoelectric constant, $E$ is electric field, $D$ is electric displacement, and $\epsilon$ is electric permittivity. Superscript ' $e$ ' refers to a ferroelectric material and subscript ' 3 ' refers to the thickness direction. Note that, $H, E$, $\chi, \sigma$ and $D$ are dependent variables that are functions of a point along its designated direction.

For the proposed nano bi-layer structure, perfect physical bonding between the FM layer and the FE layer and uniformly applied magnetic field are assumed. This external magnetic field induces the mechanical strain in the longitudinal direction that results in equal elastic displacements $(\xi)$ in both layers. The elastic displacement at a point along the longitudinal direction is related to the mechanical strain at that point by $\chi_{1}=\frac{d \xi_{1}}{d x}$.

The Newton's second law and the relation between force and stress shown in Eqs. (4) and (5) respectively, are used to explain the relation between stress and elastic displacement in Eq. (6).

$d F=M \frac{d^{2} \xi_{1}}{d t^{2}}=\rho d V \frac{d^{2} \xi_{1}}{d t^{2}}=\rho d x d y d z \frac{d^{2} \xi_{1}}{d t^{2}}$

$d F=\sigma_{1} d A=\sigma_{1} d y d z$

$\frac{\sigma_{1}}{d x} d y d z=\rho d y d z \frac{d^{2} \xi_{1}}{d t^{2}}$

where $F$ is the mechanical force, $M$ is the mass of material, $\xi_{1}$ is the elastic displacement at a point along the longitudinal direction, $V$ is the volume of the material, $A$ is the area, and $\rho$ is the material density.

The piezoelectric effect induced by the mechanical coupling generates the uniform electric field in the transverse direction across the FE layer. By substituting Eqs. (1) and (2) into Eq. (6), the relationships of strains and elastic displacements in the FM and FE layers can be expressed as

$$
\begin{gathered}
\frac{1}{s_{11}^{m}} \frac{d \chi_{1}^{m}}{d x} w \times t_{m}=\rho_{m} \times w \times t_{m} \frac{d^{2} \xi_{1}}{d t^{2}} \\
\frac{1}{s_{11}^{e}} \frac{d \chi_{1}^{e}}{d x} w \times t_{e}=\rho_{e} \times w \times t_{e} \frac{d^{2} \xi_{1}}{d t^{2}}
\end{gathered}
$$

respectively, where $t_{\mathrm{m}}$ and $t_{\mathrm{e}}$ are the thickness of FM and FE layers, respectively.

The total force of these system is obtained by combining Eqs. (7) and (8). The result is shown in Eq. (9) as 
$\frac{1}{s_{11}^{m}} \frac{d \chi_{1}^{m}}{d x} t_{m}+\frac{1}{s_{11}^{e}} \frac{d \chi_{1}^{e}}{d x} t_{e}=\left(\rho_{m} t_{m}+\rho_{e} t_{e}\right) \frac{d^{2} \xi_{1}}{d t^{2}}$.

Eq. (9) is then rearranged and simplified by defining $t$ as the total thickness or $t=t_{\mathrm{e}}+t_{\mathrm{m}}$ and $\eta$ as the thickness ratio or $\eta=\frac{t_{e}}{t_{e}+t_{m}}$. The result is expressed as

$$
\left[\frac{\eta s_{11}^{m}+(1-\eta) s_{11}^{e}}{s_{11}^{m} s_{11}^{e}}\right] \frac{d^{2} \xi_{1}}{d x^{2}}=\left[(1-\eta) \rho_{m}+\eta \rho_{e}\right] \frac{d^{2} \xi_{1}}{d t^{2}} .
$$

Since the objective of this research is to investigate the ME effect of the nano bi-layer L-T mode bar structure in high frequency environment, therefore Eq. (10) is transformed into the frequency domain as

$$
\left[\frac{\eta s_{11}^{m}+(1-\eta) s_{11}^{e}}{s_{11}^{m} s_{11}^{e}}\right] \frac{d^{2} \xi_{1}}{d x^{2}}=-\omega^{2}\left[(1-\eta) \rho_{m}+\eta \rho_{e}\right] \xi_{1},
$$

where $\omega$ is the angular frequency, which equals $2 \pi f$, and $f$ is the operating frequency.

Rewriting Eq. (11) in the second order linear differential equation form yields

$\frac{d^{2} \xi_{1}}{d x^{2}}+k^{2} \xi_{1}=0$

where $k^{2}=\omega^{2\left[(1-\eta) \rho_{m}+\eta \rho_{e}\right] s_{11}^{m} s_{11}^{e}} \frac{\eta s_{11}^{m}+(1-\eta) s_{11}^{e}}{0 .}$.

The second order linear differential equation gives a solution in a sinusoidal form as

$$
\xi_{1}(x)=A \cos (k x)+B \sin (k x),
$$

where $A$ and $B$ are constants, and $x$ is a point along the longitudinal direction.

By substituting the elastic displacement in Eq. (13) into Eqs. (1) and (2), the stresses as a function of $x$ in both layers can be expressed as

$$
\begin{aligned}
& \sigma_{1}^{m}(x)=\frac{1}{s_{11}^{m}}\left[k(B \cos (k x)-A \sin (k x))-d_{11}^{m} H_{1}\right] \\
& \sigma_{1}^{e}(x)=\frac{1}{s_{11}^{e}}\left[k(B \cos (k x)-A \sin (k x))-d_{31}^{e} E_{3}\right] .
\end{aligned}
$$

The boundary condition is then applied to determine the constant $A$ and $B$ at $x= \pm \frac{l}{2}$, where the stress is zero due to zero external force. The result shows that

$$
A=0 \text { and } B=\frac{\eta s_{11}^{m} d_{31}^{e} E_{3}+(1-\eta) s_{11}^{e} d_{11}^{m} H_{1}}{k\left[\eta s_{11}^{m}+(1-\eta) s_{11}^{e}\right] \cos (k l / 2)} \text {. }
$$

Substituting $A, B$, and Eq. (15) into Eq. (3) yields
$D_{3}=\frac{d_{31}^{e}}{s_{11}^{e}}\left[\frac{\delta d_{31}^{e} E_{3}+\beta d_{11}^{m} H_{1}}{(\delta+\beta) \cos (k l / 2)} \cos (k x)-d_{31}^{e} E_{3}\right]+\epsilon_{33} E_{3}$,

where $\delta=\eta s_{11}^{m}$ and $\beta=(1-\eta) s_{11}^{e}$

In order to calculate the ME coefficient, the evaluation of electric field across the FE layer is necessary. This is accomplished by determining the current flow through the FE layer and zeroing it out under the open circuit condition. First, the relations between the current and electric charge and the electric displacement and electric charge as shown in Eqs. (17) and (18) respectively, must be defined.

$I_{3}=\frac{d Q}{d t}$

$D_{3}=\frac{d Q}{d A}$,

where $d A=w d x$, the differential area through which the current flows.

The total current is found by substituting Eq. (18) into Eq. (17) and performing the integration as shown in Eq. (19).

$$
I_{3}=\int_{-\frac{l}{2}}^{\frac{l}{2}} \frac{d D_{3}}{d t} d A
$$

Transferring Eq. (19) to the frequency domain yields

$I_{3}=\int_{-\frac{l}{2}}^{\frac{l}{2}} i \omega D_{3} w d x=i \omega w \int_{-\frac{l}{2}}^{\frac{l}{2}} D_{3} d x$.

It can be seen that the total current, $I_{3}$, is now a function of the electric displacement, $D_{3}$, which is shown in Eq. (16). Substituting Eq. (16) intoEq. (20) thus yields

$$
I_{3}=i \omega l\left[\frac{d_{31}^{e}}{s_{11}^{e}}\left(\frac{\delta d_{31}^{e} E_{3}+\beta d_{11}^{m} H_{1}}{(\delta+\beta)} \frac{\tan \left(\frac{k l}{2}\right)}{\frac{k l}{2}}\right)-\frac{d_{31}^{e}{ }^{2}}{s_{11}^{e}} E_{3}+\epsilon_{33} E_{3}\right] .
$$

Under the open circuit condition, the current between the electrode is equal to zero and Eq. (21) is simply reduced to $\frac{\beta d_{31}^{e} d_{11}^{m}}{s_{11}^{e}(\delta+\beta)} \frac{\tan \left(\frac{k l}{2}\right)}{\frac{k l}{2}} H_{1}=\left[\frac{d_{31}^{e}{ }^{2}}{s_{11}^{e}}-\frac{\delta d_{31}^{e}{ }^{2}}{s_{11}^{e}(\delta+\beta)} \frac{\tan \left(\frac{k l}{2}\right)}{\frac{k l}{2}}-\epsilon_{33}\right] E_{3}$.

By rearranging Eq. (22), the ME coefficient, which is the ratio between the induced electric field and the applied magnetic field, can be found as shown in Eq. (23).

$$
\alpha_{31}^{H}=\frac{E_{3}}{H_{1}}=\frac{\beta d_{31}^{e} d_{11}^{m} \frac{\tan \left(\frac{k l}{2}\right)}{\frac{k l}{2}}}{d_{31}^{e}\left[\delta\left(1-\frac{\tan \left(\frac{k l}{2}\right)}{\frac{k l}{2}}\right)+\beta\right]-\epsilon_{33} s_{11}^{e}(\delta+\beta)} .
$$




\section{ME coupling behavior of Terfenol-D and PZT bi-layer bar structure}

The ME coefficient model is tested on the bi-layer of Terfenol-D and PZT. The material parameters are shown as follows. $d_{11}^{m}=11 \times 10^{-9} \mathrm{~m} / \mathrm{A}, s_{11}^{m}=17.9 \times 10^{-12} \mathrm{~m}^{2} / \mathrm{N}, \rho_{m}$ $=9,250 \mathrm{~kg} / \mathrm{m}^{3}, d_{31}^{e}=-0.6 \times 10^{-10} \mathrm{~m} / \mathrm{V}, s_{11}^{e}=1.07 \times 10^{-11}$ $\mathrm{ms}^{2} / \mathrm{kg}, \rho_{e}=7,700 \mathrm{~kg} / \mathrm{m}^{3}, \epsilon_{33}$ and $=617 \epsilon_{o}$ Colussi et al. (2017); Guo et al. (2013). The ME coupling behaviour influenced by structure dimension and operating frequency is investigated. It is found that the value of $f$ is maximum at around 1,228 at $\eta=0.19$ limit. The range of $f$ is then simply selected between 0.1 and 1,200. Fig. 2 shows the ME coefficient as a function of thickness ratio at $f l=0.1,1$, $10,100,200,500,1,000,1,100,1,200$, and $f \rightarrow 0$. It can be seen that all ME coefficient traces exhibit a parabolic-like shape with the overall highest ME coefficient at $f=1,200$. For the proposed bi-layer structure, the high frequency environment and/or long structure are then desirable in promoting the ME effect.

Fig. 3 shows the plot of the optimal thickness ratio and corresponding peak ME coefficient as a function of $f$. The optimal thickness ratio decreases and its corresponding peak ME coefficient increases with $f$. The observed exponential trend can be explained by the dependence on the $\tan (k l / 2) /(k l / 2)$ term. This result indicates the trade-off that needs to be considered in the ME sensor design. The minimum and maximum peak ME coefficients at $f l=0.1$ and 1,200 respectively, are approximately 1,756 and 5,617 $\mathrm{mV} / \mathrm{Oe} \cdot \mathrm{cm}$ with $\eta=0.43$ and 0.19 , respectively.

The mechanism responsible for the observed ME behavior is known as the magnetostrictive effect, which addresses mechanical stress and strain induced by the applied magnetic field in the FM layer. Knowing the

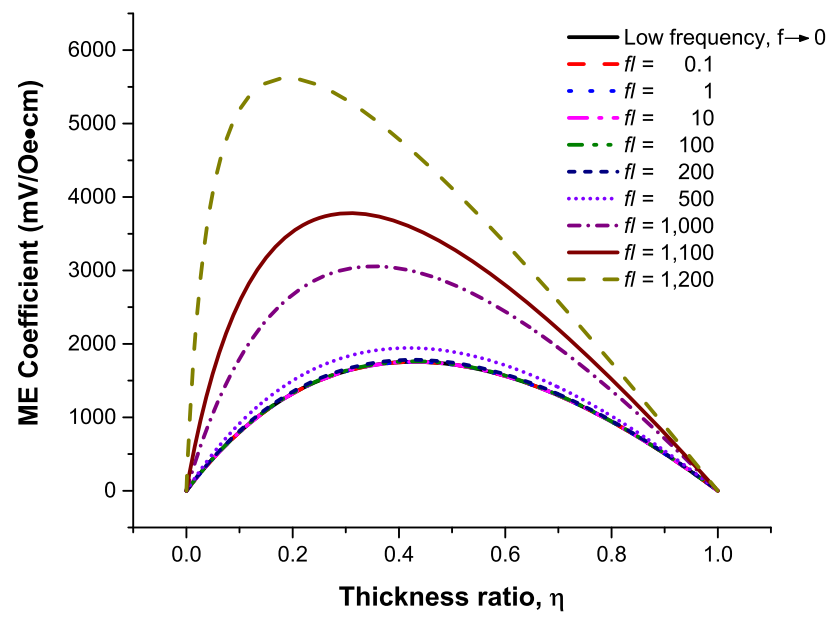

Fig. 2 The ME coefficietn as a function of thickness ratio for $f l=0.1$ 1,200

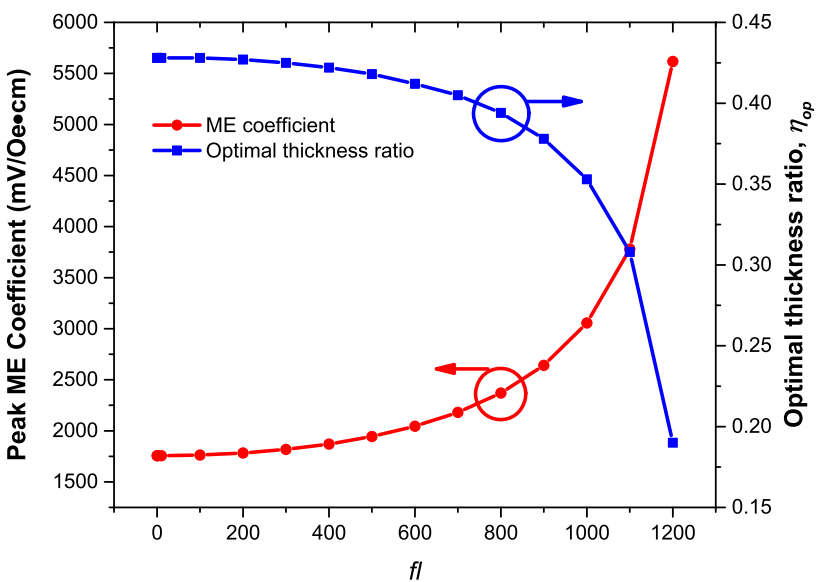

Fig. 3 The peak ME coefficient and optimal thickness ratio as a function of $f$

variation of both parameters will certainly provide insights on the ME behavior. Since FM and FE layers are perfectly bonded, the mechanical stress and strain in both layers are assumed equal. Equations (1) and (14) are then used to evaluate the stress and strain as a function of a point along the longitudinal direction as shown in Eqs. (24) and (25), respectively.

$$
\begin{aligned}
& \sigma_{1}^{m}(x)=\frac{1}{s_{11}^{m}}\left[\frac{\delta d_{31}^{e} E_{3}+\beta d_{11}^{m} H_{1}}{(\delta+\beta) \cos \left(\frac{k l}{2}\right)} \cos (k x)-d_{11}^{m} H_{1}\right] \\
& \chi_{1}^{m}(x)=\frac{\delta d_{31}^{e} E_{3}+\beta d_{11}^{m} H_{1}}{(\delta+\beta) \cos \left(\frac{k l}{2}\right)} \cos (k x)
\end{aligned}
$$

To determine the stress and strain, the average stress and strain per structure length are evaluated and can be expressed as

$$
\begin{gathered}
\bar{\sigma}_{1}^{m}=\frac{1}{l} \int_{0}^{l} \sigma_{1}^{m}(x) d x=\frac{1}{s_{11}^{m}}\left[\frac{\delta d_{31}^{e} \alpha_{31}^{H} H_{1}+\beta d_{11}^{m} H_{1}}{(\delta+\beta) \cos \left(\frac{k l}{2}\right)} \frac{\sin (k l)}{k l}-d_{11}^{m} H_{1}\right] \\
\bar{\chi}_{1}^{m}=\frac{1}{l} \int_{0}^{l} \chi_{1}^{m}(x) d x=\frac{\delta d_{31}^{e} \alpha_{31}^{H} H_{1}+\beta d_{11}^{m} H_{1}}{(\delta+\beta) \cos \left(\frac{k l}{2}\right)} \frac{\sin (k l)}{k l} .
\end{gathered}
$$

It is clearly seen that both stress and strain are linearly proportional to the applied magnetic field. To further observe their characteristics, a sample magnetic field of 190 Oe is applied to Eqs. (26) and (27). Note that, this magnetic field strength is a typical value obtained from a magnetic bit of a hard disk Choowitsakunlert et al. (2017). Figures 4 and 5 show the average stress and strain induced in the Terfenol-D as a function of $\eta$ for different $f$ value, respectively. It can be seen that their profiles are similar to that of the ME coefficient. Both stress and strain increase with $f l$ and at each $f$, reach the peak values exactly at the 


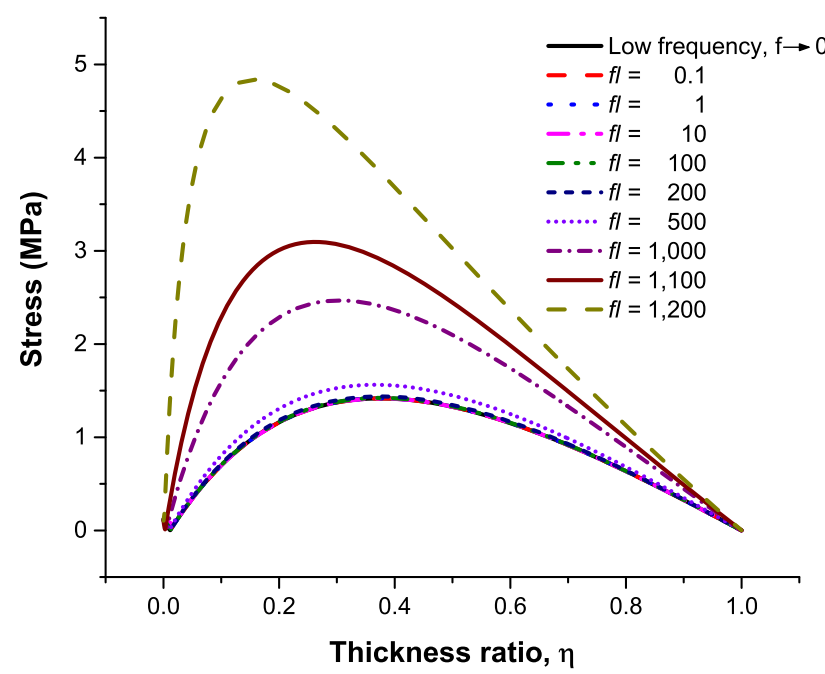

Fig. 4 The stress as a function of external magnetic field for $f l=0.1$ 1,200

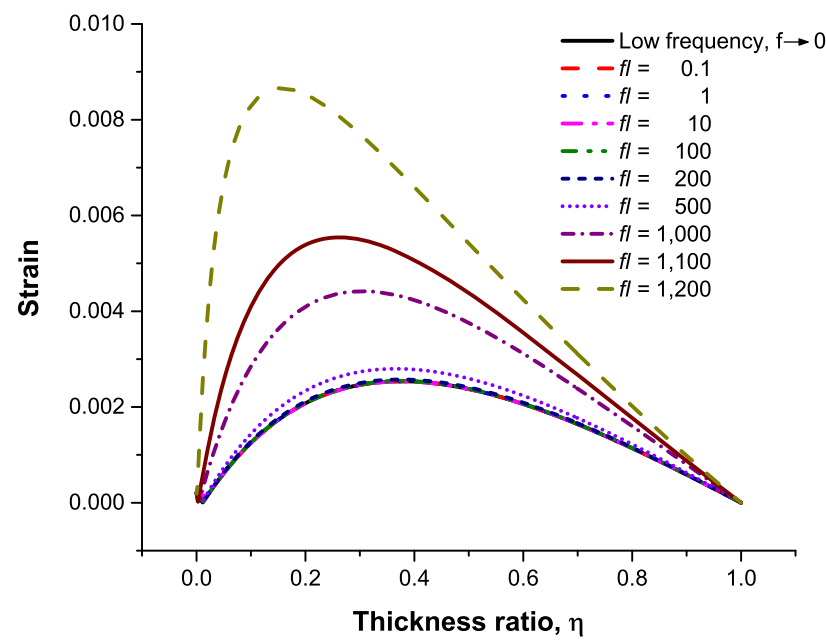

Fig. 5 The strain as a function of external magnetic field for $f=0.1$ 1,200

same thickness ratio as that of the ME coefficient. These findings are sufficient to confirm the magnetostrictive effect as a key mechanism in promoting the ME effect. The maximum and minimum stress and strain are around 4.84 and $1.42 \mathrm{MPa}$, and 0.087 and 0.0025 , with $\eta=0.19$ and 0.43 , respectively.

\section{Demotration of the nano bi-layer structure as a magnetic read head for HDD}

In this section, the demonstration of the ME nano bi-layer structure as the magnetic read sensor for the hard disk drive (HDD) is presented. The read sensor currently used in a typical HDD is the tunneling magnetoresistive (TMR) type. The TMR sensor detects a magnetic signal and changes its resistance accordingly. It requires a current to read out the output signal in forms of the voltage. The proposed structure is mechanized by direct conversion of the magnetic field into the electric field. The current is thus not required. This is believed to help lower power consumption of the HDD.

The output voltage across the FE layer can be determined by multiplying $H_{3}$ from Eq. (23) with the FE thickness as shown in Eq. (28)

$$
V_{\text {out }}=\alpha_{31}^{H} H_{1} t_{e}=\frac{\beta d_{31}^{e} d_{11}^{m} \frac{\tan \left(\frac{k l}{2}\right)}{\frac{k l}{2}} H_{1} \eta t}{d_{31}^{e}\left[\delta\left(1-\frac{\tan \left(\frac{k l}{2}\right)}{\frac{k l}{2}}\right)+\beta\right]-\epsilon_{33} s_{11}^{e}(\delta+\beta)} .
$$

The magnetic read sensor with the total thickness of $4 \mathrm{~nm}$ Vopsaroiu et al. (2008); Chong et al. (2011) is assumed. It is expected to read $2 \mathrm{Tbit} / \mathrm{in}^{2}$ areal density and still provides sufficient readback voltage. Using Eq. (28) for different $f l$ yields the voltage profiles shown in Fig. 6. The plot shows that the voltage increases linearly with the external magnetic field and at the same magnetic field, it increases with $f l$. The profile slopes are higher for higher $f$ and more prominent at $f=1,200$. At the magnetic field strength of $190 \mathrm{Oe} / \mathrm{bit}$, the output voltage is around 0.43 $\mathrm{mV}$, considerably high for the raw readback signal. All results indicate that the improvement of sensing capability can be accomplished by increasing the applied frequency or the structure length or both.

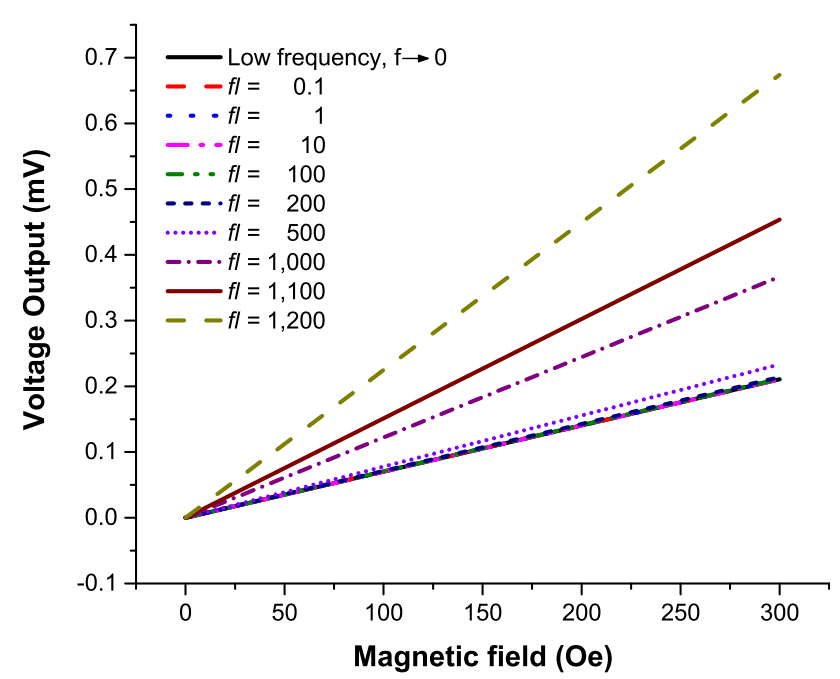

Fig. 6 The voltage output as a function of external magnetic field for $f l=0.1-1,200$ 


\section{Conclusions}

The ME coupling behaviour coefficient of the nano bi-layer L-T mode bar structure is analysed using the mathematical model of the ME that is developed in high frequency regime. The model is tested on the bi-layer of Terfenol-D/ PZT at $f l=0.1-1,200$. It is shown that all ME coefficient traces exhibit the parabolic-like shape. The optimal thickness ratio decreases exponentially and its corresponding peak ME coefficient increases exponentially with $f$. The minimum and maximum peak ME coefficients at $f=0.1$ and 1,200 respectively, are approximately 1,756 and 5,617 $\mathrm{mV} / \mathrm{Oe} \cdot \mathrm{cm}$ with $\eta=0.43$ and 0.19 , respectively. Both stress and strain profiles are similar to that of the $\mathrm{ME}$ coefficient. Stress and strain increase with $f$ and their peaks occur at the same thickness ratio as that of the ME coefficient. These findings thus confirm the magnetostrictive effect as a key mechanism responsible for the observed $\mathrm{ME}$ behavior. Then, it is found that the output voltage of the proposed structure increases linearly with the magnetic field and also increases with $f$. The demonstration of the proposed structure as the read sensor for the HDD containing a 2 Tbit/in ${ }^{2}$ areal density shows the readback voltage of $0.43 \mathrm{mV}$ at $f=1,200$. This voltage value is considerably high for a raw readback signal. All results indicate that the sensing capability can be improved by increasing the applied frequency or the structure length or both.

Acknowledgements This work has been supported by Thailand Research Fund under Research and Researchers for Industries-RRI program with the contract number PHD/58I0004. The authors would like to thank King Mongkut's University of Technology Thonburi, the University of Portsmouth, and Seagate Technology, Thailand for their equipment, software, and any valuable advices.

Open Access This article is licensed under a Creative Commons Attribution 4.0 International License, which permits use, sharing, adaptation, distribution and reproduction in any medium or format, as long as you give appropriate credit to the original author(s) and the source, provide a link to the Creative Commons licence, and indicate if changes were made. The images or other third party material in this article are included in the article's Creative Commons licence, unless indicated otherwise in a credit line to the material. If material is not included in the article's Creative Commons licence and your intended use is not permitted by statutory regulation or exceeds the permitted use, you will need to obtain permission directly from the copyright holder. To view a copy of this licence, visit http://creativecommons. org/licenses/by/4.0/.

\section{References}

Vopsaroiu M, Blackburn J, Cain M (2007) A new magnetic recording read head technology based on the magneto-electric effect. J Phys D Appl Phys 40:5027-5033

Palneedi H, Annapureddy V, Priya S, Ryu J (2006) Status and perspectives of multiferroic magnetoelectric composite materials and applications. Actuators 5:1-31

Choowitsakunlert S, Satitchantrakul T, Silpunt R (2014) A 1D analysis of nano multiferroic composites for the novel read head technology. Adv Mater Res 1052:149-154

Nan C, Bichurin M, Dong S, Viehland D, Srinivasan G (2008) Multiferroic magnetoelectric composites: Historical perspective, status, and future directions. J Appl Phys 103:031101-34

Saengow T, Satitchantrakul T, Silapunt R (2018) Investigation of Magnetoelectric Effect in the Bi-Layer Plate Structure. Solid State Phenomena 280:9-14

Wang Y, Hu J, Lin Y, Nan C (2010) Multiferroic magnetoelectric composite nanostructures. NPG Asia Materials 2:61-68

Saengow T, Silapunt R (2020) A comparative investigation of magnetoelectric characteristics of nano bi-Layer bar and plate structures. IOP Conf Series Mater Sci Eng 744:012030

Colussi M, Berto F, Razavi S, Ayatollahi M (2017) Experimental and numerical investigations of fracture behavior of magnetostrictive materials. Procedia Struct Integrity 3:153-161

Guo Q, Cao G, Shen I (2013) Measurements of Piezoelectric Coefficient d33 of Lead Zirconate Titanate Thin Films Using a Mini Force Hammer. J Vibr Acoustics 135:011003-9

Choowitsakunlert S, Silapunt R, Yokoi H (2017) A 1D study of antiferromagnetic operated on multiferroic composites in nano read head. Microsyst Technol 23:5143-5147

Vopsaroiu M, Blackburn J, Muniz-Piniella A, Cain Markys G (2008) Multiferroic magnetic recording read head technology for $1 \mathrm{Tbit} /$ in2 and beyond. J Appl Phys 103:07F506

Chong TC, Piramanayagam SN, Sbiaa R (2011) Perspectives for 10 terabits/in2 magnetic recording. J Nanosci Nanotechnol 11(3):2704-2709

Publisher's Note Springer Nature remains neutral with regard to jurisdictional claims in published maps and institutional affiliations. 